\title{
Detection of the spotted wing drosophila, Drosophila suzukii, in continental sub-Saharan Africa
}

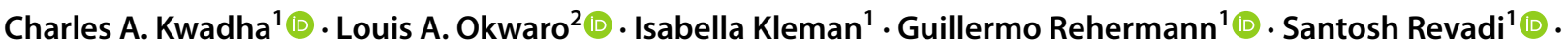

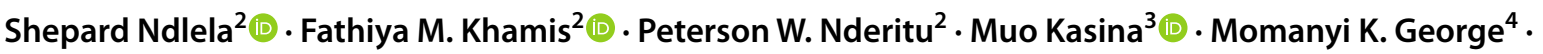

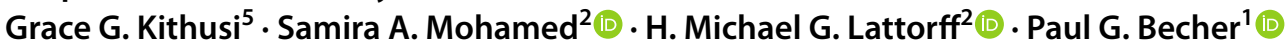

Received: 5 October 2020 / Revised: 21 December 2020 / Accepted: 5 January 2021 / Published online: 23 January 2021

(c) The Author(s) 2021

\begin{abstract}
The spotted wing drosophila, Drosophila suzukii Matsumura, is an insect pest of soft-skinned fruit, native to Eastern Asia. Since 2008, a world-wide dispersal of D. suzukii is seen, characterized by the establishment of the pest in many Asian, American and European countries. While the potential for invasion of continental Africa by D. suzukii has been predicted, its presence has only been shown for Morocco in Northern Africa. Knowledge about a possible establishment in other parts of the continent is needed as a basis for pest management. In 2019, we carried out a first survey in three counties in Kenya to monitor for the presence of $D$. suzukii using traps baited with a blend of apple cider vinegar and red wine. A total of 389 D. suzukii flies were captured in a fruit farm at Nakuru county, with more female flies being trapped than males. We confirmed the morphological identification of $D$. suzukii using DNA barcoding. In 2020, we performed a follow-up survey at 14 locations in six counties to delimit the distribution of $D$. suzukii in the main berry growing zones in Kenya. The survey indicated that so far $D$. suzukii is restricted to Nakuru county where it was initially detected. This is the first study to provide empirical evidence of $D$. suzukii in continental sub-Saharan Africa, confirming that the pest is expanding its geographic range intercontinentally. Given the high dispersal potential of $D$. suzukii, a concerted effort to develop management strategies is a necessity for containment of the pest.
\end{abstract}

Keywords Drosophilidae · Fruit fly · Global · Invasive pest · Soft fruits ·

\section{Key message}

Communicated by Antonio Biondi.

Supplementary Information The online version contains supplementary material available at https://doi.org/10.1007/s1034 0-021-01330-1.

Paul G. Becher

Paul.Becher@slu.se

1 Department of Plant Protection Biology, Swedish University of Agricultural Sciences, Box 102, 23053 Alnarp, Sweden

2 International Centre of Insect Physiology and Ecology (Icipe), P.O. Box30772-00100, Nairobi, Kenya

3 National Sericulture Research Centre, Kenya Agricultural and Livestock Research Organisation, P.O. Box 7816-01000, Thika, Kenya

4 Phytosanitary Division, KEPHIS Headquarters, Karen, P.O. Box 49592-00100, Nairobi, Kenya

5 Agriculture and Food Authority, Horticultural Crops Directorate (HCD), P.O. Box 42601-00100, Nairobi, Kenya
- Despite the global spread of Drosophila suzukii, its distribution in Africa lacks clarity.

- We carried out a monitoring study in Kenya by using traps and investigating fruit samples.

- Drosophila suzukii was captured in raspberry, strawberry blueberry and pomegranate plants.

- Morphological identification was confirmed using DNA barcoding.

- The findings confirm the presence of D. suzukii in continental sub-Saharan Africa. 


\section{Introduction}

Native to Asia, the spotted wing drosophila (SWD) Drosophila suzukii Matsumura (Diptera: Drosophilidae) was first reported as a pest in wild blueberries and cherry orchards in Shioyama Town, Japan, in 1916 (Kanzawa 1939). Since then, D. suzukii has become known as a pest of soft and stone fruits which inflicts serious economic loss to the fruit industry (Asplen et al. 2015). The loss is attributed to the serrated ovipositor of the female with which it pierces the skin of ripening fruits. In addition to the direct damage through oviposition and subsequent larval feeding, damaged fruits also get exposed to secondary infestation by other drosophilids and opportunistic pathogens (Rombaut et al. 2017).

Drosophila suzukii has nowadays been reported in many parts of the globe outside Asia. In 1980, it was reported in Hawaii and later in 2008 in the Pacific coast of California as an invasive pest (Bolda et al. 2010; Goodhue et al. 2011). Subsequently, D. suzukii has been found in other parts of the Americas (Hauser 2011; Walsh et al. 2011) as well as across European countries (Calabria et al. 2012; Ørsted and Ørsted 2019). Comparisons between selected European and North American D. suzukii populations reveal that invasions occurred through different routes as the latter populations were suggested to originate from southeast China while the former were traced to northeast China (Rota-Stabelli et al. 2020). Overall, D. suzukii shows rapid and ongoing inter-and intracontinental dispersal. Recently, D. suzukii was confirmed from samples collected in Morocco during 2017 and 2018, demonstrating presence in Northern Africa (Boughdad et al. 2020). Observations of $D$. suzukii are reported in Réunion island (IPPC 2017) and in the Comoros archipelago (Hassani et al. 2020). However, it was unknown if D. suzukii is present in continental sub-Saharan Africa.

Distribution models predict the establishment of $D$. suzukii in numerous sub-Saharan countries (Dos Santos et al. 2017) which would likely affect fruit production similar as seen in other regions. In sub-Saharan Africa, fruits generally are produced through irrigated, urban-based or highland mixed-rainfed farming systems (Costa et al. 2013; Dixon et al. 2001). While irrigated and urban-based farming systems mainly target foreign markets, rain-fed farming systems mostly supply local markets (Costa et al. 2013; Dixon et al. 2001). Moreover, unlike rain-fed farming systems which are characterized by indigenous skills and knowledge, irrigated and urban-based farming systems apply modern farming technologies, including integrated pest management (De Bon et al. 2014; Pretty and Bharucha 2015).

Generally, the global spread of $D$. suzukii is aided by international trade (Cini et al. 2014). Sub-Saharan Africa is trading commercial commodities such as fruits and vegetables, on the international market (European Union 2019; Legge et al. 2006). For example, South Africa, Cameroon, Kenya, Ghana and Ethiopia are exporting off-season and tropical fruits to both regional and international trade partners (Hoffmann and Vossenaar 2008).

Kenya, as a strategically located country, provides a bridge, through its port of Mombasa, that allows for crossborder movement of commodities to the neighboring landlocked countries including Uganda, Burundi, Rwanda and South Sudan (Sebuny 2015). Consequently, Kenya and the neighboring partner countries are exposed to a high risk of invasion by economically significant insect pests. A prime example of such an invasion is represented by Bactrocera species, such as Bactrocera dorsalis (Hendel) (Diptera; Tephritidae), which originates from Asia and was first recorded in Kenya with subsequent reports of its establishment in other Eastern, Central and Southern African countries (Drew et al. 2005; Lux et al. 2003). As of 2018, Kenya was importing strawberries, raspberries, blackberries, gooseberries, cherries and plums, which all are known as D. suzukii host fruits (International Trade Centre 2019). Of the fruit-importing countries, South Africa, Italy, Egypt, Spain, Chile and India the four non-African countries have confirmed the presence of $D$. suzukii (International Trade Centre 2019; CABI 2019; Kenis et al. 2016). Introduction of D. suzukii might lead to a permanent invasion and dispersal of the pest in sub-Saharan Africa. Through inductive modeling, Dos Santos et al. (2017) previously showed that southwestern Kenya and bordering countries (Uganda and Tanzania) have suitable abiotic conditions that would support D. suzukii establishment.

Evidently, the above-mentioned factors provided a backdrop on which we hypothesized that $D$. suzukii might already have been introduced into continental sub-Saharan Africa and be present in soft and stone fruit cultivating regions. To address this hypothesis, we carried out a monitoring survey during 2019 and 2020. We report the detection but apparently restricted distribution of D. suzukii in Kenya. Species identity was confirmed using morphological features together with DNA barcoding of the mitochondrial cytochrome oxidase subunit I (COI).

\section{Material and Methods}

\section{Detection survey in 2019}

To monitor for potential invasion of $D$. suzukii in Kenya, a survey was carried out during the dry season, from 13th to July 20th, 2019, in three counties; Nairobi county (farm: Duduville campus of the International Centre of Insect Physiology and Ecology (icipe), $1^{\circ} 22^{\prime} \mathrm{S}, 36^{\circ} 89^{\prime} \mathrm{E}$, about 
1, $600 \mathrm{~m}$ above sea level, hereafter masl); Kiambu county (farm: Sasini estate $1^{\circ} 16^{\prime} \mathrm{S}, 36^{\circ} 89^{\prime} \mathrm{E}$, about 1,700 masl) and Nakuru county (farm: Longonot farm $0^{\circ} 83^{\prime} \mathrm{S}, 36^{\circ} 38^{\prime} \mathrm{E}$, about 1,935 masl). We used the Leaflet package in $\mathrm{R} v 2.0 .2$ (Cheng et al. 2018) to show the locations on a map (Fig. S1). Brief descriptions of icipe, Sasini estate and Longonot farm are given in the supplementary material. In each of the farm, 10 traps (1 trap/site, see supplementary for details; Fig. S2) of $60-\mathrm{mL}$ volume filled with $40 \mathrm{~mL}$ of a blend of apple cider vinegar and red wine (20:80 v:v) were used (Huang et al. 2017; Landolt et al. 2012). At icipe and Sasini, we monitored crops cultivated in a mixed-cropping system. At Longonot, we sampled raspberries (Rubus idaeus), strawberries (Fragaria ananassa), blueberries (Vaccinium corymbosum) and pomegranates (Punica grantum) (Table S1) grown in separate tunnels (Fig. S3). Two traps were placed per tunnel. At the time of the survey, fruit ripening was more advanced in raspberries than in the other crops. After a week, the captured insects were collected and inspected for the presence of $D$. suzukii by morphological features using a Zeiss microscope (Göttingen, Germany) fitted with an Axiocam 105 color microscope camera (Göttingen, Germany). Generally, the adults were about $2-3 \mathrm{~mm}$ long, having red eyes with brown thorax and abdomen, with unbroken bands. The males were characterized by a dark spot on each wing (Fig. S4a) and a sex comb on the first and second segment of the foreleg with 3-6 teeth running parallel to the feet (Fig. S4b). Females were identified based on the distinctive ovipositor with a pronounced serration that is different from $D$. subpulchrella (Atallah et al. 2014; Vlach 2010) (Fig. S4c).Through a weather information provider (Visual Crossing 2020), daily data on temperature, relative humidity and precipitation were extracted from nearest accessible weather stations in Nairobi, Kiambu and Naivasha (Nakuru) representing the respective survey sites (Table S3). In a follow-up survey in 2020 (see below), weather data were recorded in Laikipia, Murang'a, Nyandarua and Nyeri (Table S4).

\section{Follow-up survey in 2020}

As it was unclear whether or not the pest had established and dispersed since its detection in Longonot in 2019, we carried out a follow-up survey between May 20 and June 11, 2020. A total of 11 farms and 3 roadsides distributed in major berry growing areas including Longonot farm (Nakuru county), Kiambu, Nyandarua, Murang'a, Nyeri and Laikipia counties were surveyed (Fig. S1 and Table S2). Roadsides have earlier been shown as suitable sites to detect $D$. suzukii (Lengyel et al. 2015). Baited Drososan traps (Koppert Biological Systems, the Netherlands) were set up at 25 sites (one trap per site) at the 14 locations (Table S2). Fruits monitored at the farms were cultivated in tunnels. After 5-7 days, traps were collected for sorting and identification at icipe. The number of caught flies was computed as daily catches of flies per trap. Like in the 2019 survey, traps were set up and collected once.

Samples of D. suzukii from both the 2019 and 2020 surveys are stored in $70 \%$ ethanol at icipe and are accessible for future reference, through the code SWD-Longonot-07-2019.

\section{Survey on infestation of berries by $D$. suzukii}

To assess host infestation, we sampled ripe raspberries, strawberries, blackberries (Rubus fruticosus) and blueberries grown at 11 farms at different locations. The specific types of fruit grown and investigated at different farms are provided in Table S2. For each type of fruit investigated at a respective farm, at least $0.2 \mathrm{~kg}$ of berries was sampled. Each sample consisted of berries that were handpicked from the plants and berries that had fallen to the ground. With the exception of $R$. idaeus and V. corymbosum at Longonot, which were collected at two dates, fruits at other farms were collected once. Collected berries were kept separately by type in 2-L plastic containers (Kenpoly Manufacturers Ltd, Nairobi, Kenya), covered with lids fitted with fine netting material. Samples were transported to the laboratories at the Animal Rearing and Containment facility at icipe. Sample weight was measured using an electrical balance (KERN PCB 2500, KERN \& Sohn GmbH Ziegelei Balingen, Germany). Developing larvae were protected from drowning in excess juice by lining each container with four layers of Velvex premium kitchen towels (Chandaria Industries Ltd, Nairobi, Kenya) which were exchanged twice a day.

Pupae were collected, transferred into a Petri-dish lined with moistened paper towel and kept in a Perspex cage $(0.2 \times 0.2 \times 0.2 \mathrm{~m})$ fitted with a fine mesh sleeve. Emerging adults of $D$. suzukii were counted and recorded as flies per kilogram of fruit.

\section{Molecular identification}

We extracted the genomic DNA from eight flies $(n=4$ per sex) caught in 2019 using phenol-chlorofom (Sambrook and Russell 2006) and from three flies ( 2 males and 1 female) that emerged from incubated berries in 2020 using Isolate II Genomic DNA kit. For polymerase chain reaction (PCR) amplification, we used $D$. suzukii specific primers to amplify the barcode region of the cytochrome oxidase I (COI) for 2019 samples and general primers (Hajibabaei et al. 2006) for 2020 samples. Amplified fragments were purified and bidirectionally sequenced at Macrogen Europe BV (Meibergreef, Amsterdam, the Netherlands). Following visual inspection and manual trimming of the DNA sequence electropherograms, we aligned forward and reverse sequences using ClustalW algorithm (Bioedit v. 7.2.5) (Hall 1999). The representative sequences were aligned to reference 
sequences from the National Center for Biotechnology Information (NCBI). Additionally, we compared the sequences with those of closely related species and generated a maximum likelihood phylogenetic tree (Fig. S5) with 1000 bootstraps using MEGA X v 10.0.5 (Kumar et al. 2018). To generate the tree, we aligned the sequences using the default settings of MUSCLE algorithm in MEGA X. The resultant alignment was used to predict the best model for constructing a phylogenetic tree using Tamura-Nei model (Tamura and Nei 1993) which had the lowest Bayesian Information Criterion (BIC $=4561.79)$. All the sequences submitted to GenBank can be accessed through: accession MN689051MN689058 for 2019 samples, and accession MT966718, MT975699 and MT981339 for 2020 samples. More details are provided in the supplementary material.

\section{Results}

Out of the 30 sites at the three locations monitored in the 2019 survey, D. suzukii was detected at ten sites at Longonot farm, Nakuru county. Flies were first identified by morphological features. We confirmed the morphological identification of D. suzukii through COI DNA fragment amplification from genomic DNA, followed by direct sequencing and BLAST searches. The resulting sequences (Table S5a) were aligned to reference sequences from NCBI compared with publicly available data on GenBank and yielded a similarity score of $100 \%$ with $D$. suzukii samples from Switzerland
(Accession number MG605095.1), Turkey (Accession number MK435596.1) and Italy (Accession number KJ671599.1) (Kuyulu et al. 2019) (Fig. S5). A total of 389 D. suzukii flies were captured in the 7-day period of the survey, in which females accounted for $62.0 \%$ (95\% confidence interval of $56.9-66.8 \%$, for binomial distribution) of the total catches. In detail, with the two traps per plot, 130 and 127 D. suzukii flies (80 and 78 females, respectively) were trapped in the two raspberry plots, 60 flies ( 47 females) in the strawberry plot, 37 flies (15 females) in the blueberry plot and 35 flies (21 females) in the pomegranate plot (Fig. 1). Other species of Diptera, Coleoptera and Hymenoptera were captured at Longonot, Sasini and icipe; these specimens were stored but not further identified. In Longonot, D. suzukii represented $60.8 \%$ of all the captured drosophilids.

In the follow-up survey in 2020, D. suzukii was detected in Nakuru county but only at Longonot farm where we had trapped flies already in 2019. From the monitoring traps, 358 D. suzukii flies were captured, in which 257, 63 and 38 flies were caught in raspberries, blueberries and strawberries, respectively. Moreover, 799 adults of $D$. suzukii emerged from incubated fruit, of which raspberries had the highest number of flies (782, compared to 14 and 3 flies that emerged from blueberries and strawberries, respectively). Drosophila suzukii was neither observed in traps nor emerged from incubated fruits collected at berry growing areas in Kiambu, Nyandarua, Murang'a, Nyeri and Laikipia counties. We further confirmed the identity of $D$. suzukii captured in the 2020 survey through morphological and molecular
Fig. 1 Drosophila suzukii monitoring results from a fruit farm in Kenya. Radial plots illustrate the mean number of D. suzukii, other drosophilids, other Dipterans, Hymenopterans and Coleopterans per trap caught across four different fruit crops in 2019. The circular grid lines of each radial plot represent 20 captured individuals belonging to different insect categories listed above

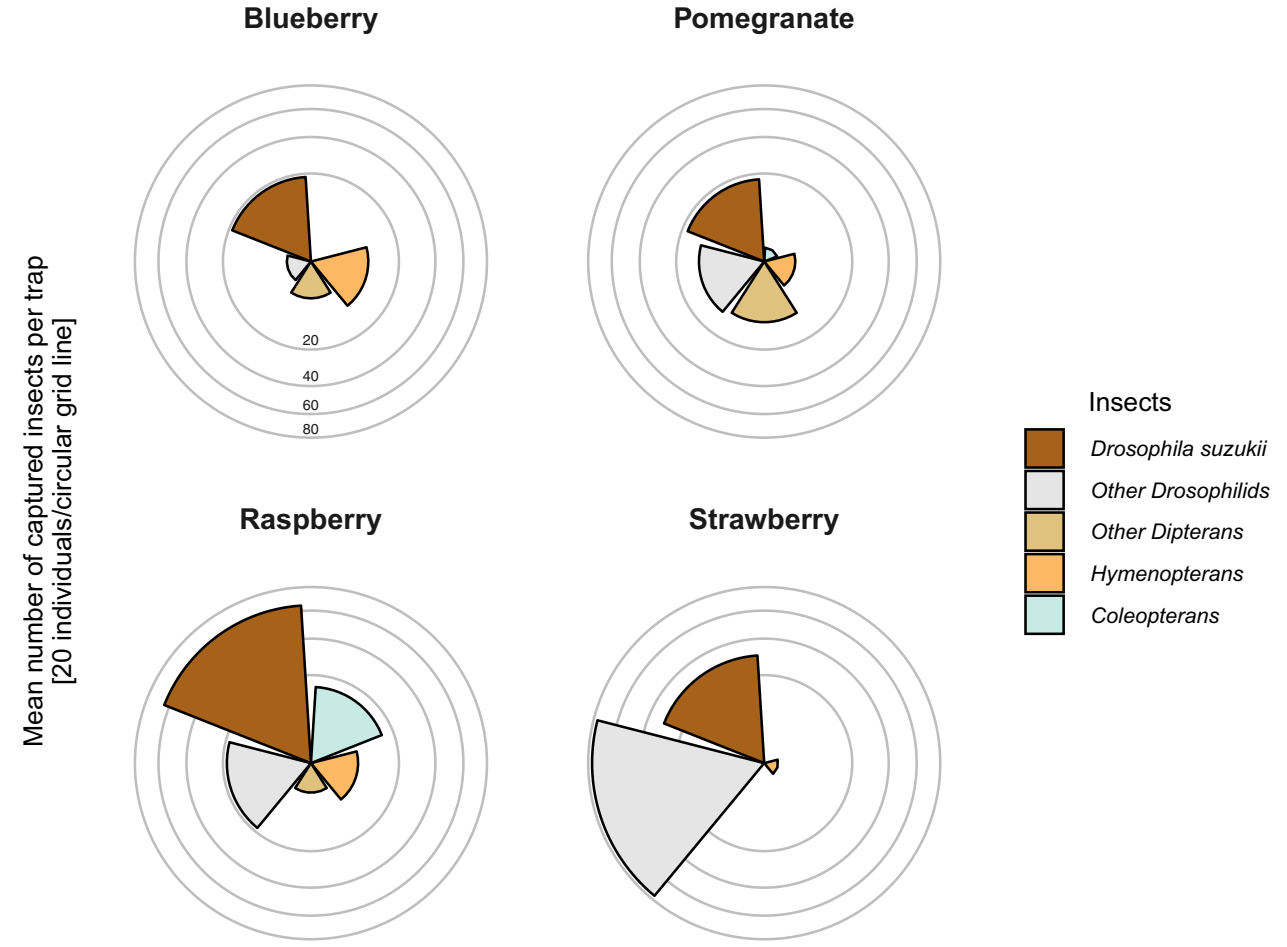


identification. The resulting sequences (Table S5b) gave a similarity score of $94-100 \%$ with reference sequences in GenBank (MK435596.1 and MK801757.1).

\section{Discussion}

In the last decade, D. suzukii has spread from its native region in Asia to America and Europe, and the possibility for further dispersal to continental Africa was predicted (Dos Santos et al. 2017). Recently, the presence of $D$. suzukii was reported in Morocco, North Africa (Boughdad et al. 2020). Through monitoring, we show the presence but apparently restricted distribution of $D$. suzukii in Kenya, sub-Saharan Africa. Morphological examination and molecular confirmation by $C O I$ barcoding were used to prove identification at the species level.

During 2019, we captured D. suzukii in Longonot, a commercial farm growing blueberries, strawberries, raspberries and pomegranates, fruits which are known to support the development of the flies (Arnó et al. 2016; Wang et al. 2019). Drosophila suzukii was captured in plots of all different fruits. The establishment of invasive $D$. suzukii at Longonot farm was confirmed in 2020 where the pest was found both in monitoring traps and sampled fruit. Traps in raspberry tunnels caught most $D$. suzukii in 2019 and raspberries were more infested than blueberries and strawberries in 2020. This suggests that $D$. suzukii infestation differs across fruits and that raspberry is the most susceptible crop to $D$. suzukii at Longonot farm. Oviposition and infestation are known to differ among host species with raspberries representing one of the preferred fruits (Burrack et al. 2013; Lee et al. 2011). Moreover, more ripe berries were observed in plots with raspberries compared to plots with other types of fruits. Drosophila suzukii prefers ripe to unripe berries (Karageorgi et al. 2017; Keesey et al. 2015) and captures of $D$. suzukii across orchards in close proximity are known to fluctuate depending on fruit phenology (Lee et al. 2015; Wang et al. 2016). In the 2019 survey, we did not investigate the infestation of fruit. However, damage by unspecified insect pests was occurring in the farm as was reported by the field manager. The detection of $D$. suzukii since 2019 and the emergence of the pest from incubated fruits collected during the 2020 survey provide a likely explanation for the reported insect damage. At Longonot, the mixed production of different crops with successive availability of ripe fruits throughout the season, as well as the practice of drip irrigation and cultivation in tunnels, might provide most suitable microhabitats facilitating $D$. suzukii establishment at the farm (Diepenbrock and Burrack 2017; Khaliq et al. 2014; Toxopeus et al. 2016).

Despite the availability of host fruits at the farms that represent 11 out of the 14 locations surveyed in 2020, D. suzukii was only found at Longonot farm. This, in consequence, does not allow conclusions about the presence or absence of the pest apart from Longonot. Repetitive detection of $D$. suzukii at Longonot proves the validity of our monitoring technique and suggests that the fly would have been detected if present. Drosophila suzukii has been shown to disperse over long distances (Tait et al. 2018). The establishment at Longonot implies the possibility of an area-wide dispersal if $D$. suzukii is not contained. In the recent past, invasive pest species of the genus Bactrocera have caused substantial losses to Africa's fruit industry (Drew et al. 2005; Ekesi et al. 2016; Lux et al. 2003). In Europe and America, D. suzukii infestation has caused huge yield losses and costs exceeding $\$ 100$ million per fruit per year (De Ros et al. 2013; Mazzi et al. 2017; Walsh et al. 2011), and therefore, Kenya and other African countries are likely to experience similar economic impact by the newly found pest. There has been a growing interest amongst small-scale farmers in Kenya, to grow soft fruits such as plums (Mbora et al. 2008) and berries. In the neighboring country, Ethiopia, strawberry cultivation is on the rise with small- and medium-scale growers adopting the practice. The detection and establishment of $D$. suzukii are a threat to the potential expansion of berry cultivation and trade in the region. Therefore, our findings serve a two-front wake-up call to stakeholders of the Kenyan and regional fruit industry. It calls for continuous region-wide monitoring to establish the extent of $D$. suzukii distribution, from which management programs can be defined and executed (Kenis et al. 2016).

In addition to monitoring, other measures have been proved as important tools to control D. suzukii and should support the development of integrated management practices (IPM) in Kenya and other African countries. Informing growers about the appearance of $D$. suzukii and how to recognize specific damage and the pest itself is a most urgent task. Self-made traps similar to that applied in our study (Fig. S2) could facilitate on-farm monitoring. Directed sanitary measures on farms should be implemented to reduce development of $D$. suzukii, e.g., in decaying fruit left on the plant or fallen to the ground (Haye et al. 2016). Further, adjustments in cultivation practice such as selection of less susceptible varieties or harvesting at an early stage of ripening before berries become soft and more likely to be infested should be considered for the coming growing seasons (Hampton et al. 2014; Lee et al. 2016). Another measure that could be applicable to farmers is the use of netting to reduce the entering of $D$. suzukii into tunnels or to cover and protect crops such as blueberries (Del Fava et al. 2017). Insecticides have been intensively studied and are applied in conventional and organic production (Van Timmeren and Isaacs 2013). For example, spinosad is one of the widely used insecticides for control of $D$. suzukii and even used in organic production (Bruck et al., 2011). 
Recent studies increase the general concern about insecticide resistance in $D$. suzukii advocating the application of sustainable pest control methods (Gress and Zalom 2019). Biological control is a promising route to reduce $D$. suzukii infestation using natural enemies and insect pathogens (Becher et al. 2018; Biondi et al 2020; Daane et al. 2016; Gabarra et al. 2015; Lee et al. 2019; Wolf et al. 2020). Findings on the specificity of parasitoids with respect to Drosophila host species, different fruit species as well as fruit ripeness give grounds for hope toward the development of biological control (Girod et al 2018; Wolf et al. 2020; Biondi et al 2020; Seehausen et al 2020). In our trap captures, nontargeted Coleopterans and Hymenopterans were recorded but not identified. Previously, predatory Coleopterans and parasitic Hymenopterans were reported in D. suzukii traps and monitoring (Wang et al. 2016; Wolf et al. 2018). The possible existence of natural enemies in the area warrants follow-up studies. Generally, D. suzukii is not limited to sites of fruit production but is often found in forests or unmanaged areas which emphasizes the need for area-wide and long-term control strategies exploiting approaches such as biological control or the sterile insect technique (Haye et al. 2016; Seehausen et al. 2020; Nikolouli et al. 2020).

The detection of $D$. suzukii at only one location indicates that the distribution of D. suzukii in Kenya still is restricted and the invasion is at an early stage. New pest invasions are characterized by an arrival and an establishment phase that generally allow management to interfere with pest dispersal (Kolar and Lodge 2001; Liebhold and Tobin 2008). Overall, the combination of different measures such as monitoring, chemical, biological and cultural management, combined with preventive cultural practice, provides a toolbox to develop a sustainable IPM approach at local and landscape scale (Cini et al. 2012; Del Fava et al. 2017; Haye et al. 2016), targeting D. suzukii as a new pest in sub-Saharan Africa.

Dos Santos et al. (2017) had predicted that Tanzania offers a larger region suitable for $D$. suzukii establishment than Kenya, suggesting that while this is the first record of the pest in continental sub-Saharan Africa, a region-wide monitoring program is required to obtain a better overview on the current status of D. suzukii occurrence. Previous studies established multiple invasions in other infested countries (Carvajal and Markow 2010; De la Vega et al. 2020; Lavrinienko et al. 2017). It would be intriguing to study population genetics of the Kenyan population and establish the possible nature of the invasion. Clearly, more information is needed about the distribution and phenology of D. suzukii in Africa, potential host fruits on farms and in the wild, and the occurrence of natural enemies. Cost estimations on the economic impact and control programs in Kenya and other African countries would be helpful to guide future strategies for adequate management. Altogether, understanding the population dynamics of $D$. suzukii in the local ecological, environmental, landscape and horticultural context will provide a benchmark for successful management.

In conclusion, by using traps we showed the presence of D. suzukii at Longonot farm (Nakuru county) in Kenya. Through host incubation in the laboratory, we confirmed infestation of the berries collected from the farm. We have through morphological examination and DNA barcoding confirmed the identity of the collected flies. Altogether, $D$. suzukii is present in Kenya but was only detected in one out of six monitored counties suggesting that the pest is at an early phase of invasion and might be absent in the main host-fruit production areas of Kenya. Our findings add to the growing list of countries recently invaded by $D$. suzukii, illustrating the exceptionally fast and ongoing geographical range expansion of this pest insect. Drosophila suzukii has a high potential of dispersal, which necessitates quick and concerted efforts to prevent further distribution in the region.

\section{Author contribution}

CAK, IK, GR and PGB conceived the idea. CAK, IK, GR, SN, MK, SAM, PN and PGB designed the experiments. CAK, SN, SAM, PWN, MK, MKG and GGK conducted the survey. CAK and SN did morphological identification. LAO, FMK, SR and HMGL did molecular identification and analysis. MK assayed fruit incubation. CAK and GR analyzed the trap capture results. CAK and PGB wrote the manuscript with contributions by SR, SN, SAM and FMK. All authors read and approved the manuscript.

Acknowledgements We thank Sunday Ekesi, Ylva Hillbur and Segenet Kelemu for their advice and support to accomplish this study and Robinson Okoth Kisero and Evans Odhiambo for their support during the field work. We are also thankful to Levi Odhiambo Ombura for assistance with molecular characterization of the flies reared from fruits, Mary Guantai from KEPHIS JKIA and Joseph Mulwa from KALRO Kabete for insect identification, Sebastian Larsson-Herrera and Adam Flöhr (statistics@slu) for assistance in R and Sharon Hill for comments and language edits. We are grateful to berry famers and the Longonot management for collaboration to access their farms. This work was supported by the Gösta och Anna-Birgit Henrikssonss foundation, the Swedish Research Council Formas (Grant 2015-1221) and the Wallenberg Foundation (travel grant), the SLU Platform Plant Protection and the SLU Centre for Biological Control (CBC); the Norwegian Agency for Development Cooperation, the Section for research, innovation, and higher education (Grant No. RAF-3058 KEN-18/0005) and the Government of Kenya through the Horticulture Research Fund (Grant to KALRO). We would also like to acknowledge the core financial assistance to icipe by UK's Foreign, Commonwealth and Development Office (FCDO); the Swedish International Development Cooperation Agency (Sida); the Swiss Agency for Development and Cooperation (SDC); the Federal Democratic Republic of Ethiopia; and the Government of the Republic of Kenya. The views expressed herein do not necessarily reflect the official opinion of the donors. 
Funding Open Access funding provided by Swedish University of Agricultural Sciences.

\section{Compliance with ethical standards}

Conflict of interest The authors declare that they have no conflict of interest.

Human and animal rights This study did not involve any human or animal (vertebrate) subject.

Informed consent Informed consent was obtained from all individual participants included on the study.

Open Access This article is licensed under a Creative Commons Attribution 4.0 International License, which permits use, sharing, adaptation, distribution and reproduction in any medium or format, as long as you give appropriate credit to the original author(s) and the source, provide a link to the Creative Commons licence, and indicate if changes were made. The images or other third party material in this article are included in the article's Creative Commons licence, unless indicated otherwise in a credit line to the material. If material is not included in the article's Creative Commons licence and your intended use is not permitted by statutory regulation or exceeds the permitted use, you will need to obtain permission directly from the copyright holder. To view a copy of this licence, visit http://creativecommons.org/licenses/by/4.0/.

\section{References}

Arnó J, Solà M, Riudavets J, Gabarra R (2016) Population dynamics, non-crop hosts, and fruit susceptibility of Drosophila suzukii in Northeast Spain. J Pest Sci 89:713-723

Asplen MK et al (2015) Invasion biology of spotted wing Drosophila (Drosophila suzukii): A global perspective and future priorities. J Pest Sci 88:469-494

Atallah J, Teixeira L, Salazar R, Zaragoza G, Kopp A (2014) The making of a pest: the evolution of a fruit-penetrating ovipositor in Drosophila suzukii and related species. Proc R Soc B 281:20132840

Becher PG, Jensen RE, Natsopoulou ME, Verschut V, Henrik H (2018) Infection of Drosophila suzukii with the obligate insect-pathogenic fungus Entomophthora muscae. J Pest Sci 91:781-787

Biondi A, Wang X, Daane KM (2020) Host preference of three Asian larval parasitoids to closely related Drosophila species: implications for biological control of Drosophila suzukii. J Pest Sci. https ://doi.org/10.1007/s10340-020-01272-0

Bolda MP, Goodhue RE, Zalom FG (2010) Spotted wing drosophila: potential economic impact of a newly established pest. Agric Resour Econ Update, Univ Calif, Giannini Found 13:5-8

Boughdad A, Haddi K, Bouazzati AE, Nassiri A, Tahiri A, Anbri CE, Eddaya T, Zaid A, Biondi A (2020) First record of the invasive spotted wing Drosophila infesting berry crops in Africa. J Pest Sci. https://doi.org/10.1007/s10340-020-01280-0

Bruck DJ et al (2011) Laboratory and field comparisons of insecticides to reduce infestation of Drosophila suzukii in berry crops. Pest Manag Sci 67:1375-1385

Burrack HJ, Fernandez GE, Spivey T, Kraus DA (2013) Variation in selection and utilization of host crops in the field and laboratory by Drosophila suzukii Matsumara (Diptera: Drosophilidae), an invasive frugivore. Pest Manag Sci 69:1173-1180
CABI (2019) Drosophila suzukii. In: Invasive Species Compedium. CAB International, Wallingford

Calabria G, Máca J, Bächli G, Serra L, Pascual M (2012) First records of the potential pest species Drosophila suzukii (Diptera: Drosophilidae) in Europe. J Applied Entom 136:139-147

Carvajal JI, Markow T (2010) Genetic diversity of Drosophila suzukii in San Diego. Dros Inf Serv 93(12):223-303

Cheng J, Karambelkar B, Xie Y (2018) Leaflet: Create Interactive Web Maps with the JavaScript "Leaflet" Library. R package version 2:2

Cini A, Ioriatti C, Anfora G (2012) A review of the invasion of Drosophila suzukii in Europe and a draft research agenda for integrated pest management. Bull Insectol 65:149-160

Costa S, Crovetto G, Bocchi S (2013) Family Farming in Africa: Overview of good agricultural practices in SSA University of Milan, ISBN:978-988

Daane KM et al (2016) First exploration of parasitoids of Drosophila suzukii in South Korea as potential classical biological agents. J Pest Sci 89:823-835

De Bon H, Huat J, Parrot L, Sinzogan A, Martin T, Malezieux E, Vayssieres J-F (2014) Pesticide risks from fruit and vegetable pest management by small farmers in sub-Saharan Africa. J Agron Sustain Develop 34:723-736

De Ros G, Anfora G, Grassi A, Ioriatti C (2013) The potential economic impact of Drosophila suzukii on small fruits production in Trentino (Italy). IOBC-WPRS Bull 91:317-321

De la Vega G, Corley JC, Soliani C (2020) Genetic assessment of the invasion history of Drosophila suzukii in Argentina. J Pest Sci 93:63-75

Del Fava E, Ioriatti C, Melegaro A (2017) Cost-benefit analysis of controlling the spotted wing drosophila (Drosophila suzukii (Matsumura)) spread and infestation of soft fruits in Trentino, Northern Italy. Pest Manag Sci 73:2318-2327

Diepenbrock L, Burrack H (2017) Variation of within-crop microhabitat use by Drosophila suzukii (Diptera: Drosophilidae) in blackberry. J Appl Entomol 141:1-7

Dixon JA, Gibbon DP, Gulliver A (2001) Farming systems and poverty: improving farmers' livelihoods in a changing world. Food Agricu Org

Dos Santos LA, Mendes MF, Krüger AP, Blauth ML, Gottschalk MS, Garcia FRJPo, (2017) Global potential distribution of Drosophila suzukii (Diptera, Drosophilidae). PLoS ONE 12(3):e0174318

Drew R, Tsuruta K, White I (2005) A new species of pest fruit fly (Diptera: Tephritidae: 'Dacinae) from Sri Lanka and Africa. African Entomol 13:149-154

Ekesi S, De Meyer M, Mohamed SA, Virgilio M, Borgemeister C (2016) Taxonomy, ecology, and management of native and exotic fruit fly species in Africa. Ann Review of Entomol 61:219-238

European Union (2019) Agri-Food Trade Statistical Factsheet. Directorate-General for Agriculture and Rural Development Unit. https ://ec.europa.eu/info/sites/info/files/food-farming-fisheries/farmi ng/documents/agrifooafrica-all-countries_en.pdf. Accessed 14-02 2020

Gabarra R, Riudavets J, Rodríguez GA, Pujade-Villar J, Arnó J (2015) Prospects for the biological control of Drosophila suzukii. Biocontrol 60:331-339

Girod P, Lierhmann O, Urvois T, Turlings TC, Kenis M, Haye T (2018) Host specificity of Asian parasitoids for potential classical biological control of Drosophila suzukii. J Pest Sci 91:1241-1250

Goodhue RE, Bolda M, Farnsworth D, Williams JC, Zalom FG (2011) Spotted wing drosophila infestation of California strawberries and raspberries: Economic analysis of potential revenue losses and control costs. Pest Manag Sci 67:1396-1402

Gress BE, Zalom FG (2019) Identification and risk assessment of spinosad resistance in a California population of Drosophila suzukii. Pest Pest Manag Sci 75:1270-1276 
Hajibabaei M, Janzen DH, Burns JM, Hallwachs W, Hebert PD (2006) DNA barcodes distinguish species of tropical Lepidoptera. PNAS 103:968-971

Hall TA BioEdit: a user-friendly biological sequence alignment editor and analysis program for Windows 95/98/NT. In: Nucleic acids symposium series, 1999. vol 41. [London]: Information Retrieval Ltd., c1979-c2000., pp 95-98

Hampton E, Koski C, Barsoian O, Faubert H, Cowles RS, Alm SR (2014) Use of early ripening cultivars to avoid infestation and mass trapping to manage Drosophila suzukii (Diptera: Drosophilidae) in Vaccinium corymbosum (Ericales: Ericaceae). J Econ Entomol 107:1849-1918

Hassani IM et al (2020) First occurrence of the pest Drosophila suzukii (Diptera: Drosophilidae) in the Comoros Archipelago (Western Indian Ocean). African Entomol 28:78-83

Hauser M (2011) A historic account of the invasion of Drosophila suzukii (Matsumura)(Diptera: Drosophilidae) in the continental United States, with remarks on their identification. Pest Manag Sci 67:1352-1357

Haye T et al (2016) Current SWD IPM tactics and their practical implementation in fruit crops across different regions around the world. J Pest Sci 89:643-651

Hoffmann U, Vossenaar R (2008) Implications for exports of fresh fruit and vegetables from sub-Saharan Africa. UNCTAD, United Nations, Geneva

Huang J, Gut L, Grieshop M (2017) Evaluation of food-based attractants for Drosophila suzukii (Diptera: Drosophilidae). Environ Entomol 46:878-884

International Trade Centre (2019) Trade statistics for international business development Monthly, quarterly and yearly trade data. Import \& export values, volumes, growth rates, market shares https://www.trademap.org/Index.aspx

IPPC (2017) Drosophila suzukii present à Tahiti and Moorea. Official Pest Reports (PYF-10/2) - French Polynesia. Food and Agriculture Organization of the United Nations. https://www.ippc.int/ en/countries/french-polynesia/pestreports/2017/07/drosophila -suzukii-present-a-tahiti-moorea/. Accessed 08, November 2019

Kanzawa T (1939) Studies on Drosophila suzukii Mats. Yamanashi Agricultural Experimental Station, Kofu, Review of Applied Entomology

Karageorgi M et al (2017) Evolution of multiple sensory systems drives novel egg-laying behavior in the fruit pest Drosophila suzukii. Curr Biol 27:847-853

Keesey IW, Knaden M, Hansson BS (2015) Olfactory specialization in Drosophila suzukii supports an ecological shift in host preference from rotten to fresh fruit. J Chem Ecol 41:121-128

Kenis M et al (2016) Non-crop plants used as hosts by Drosophila suzukii in Europe. J Pest Sci 89:735-748

Khaliq A, Javed M, Sohail M, Sagheer M (2014) Environmental effects on insects and their population dynamics. J Entomol Zool Stud 2:1-7

Kolar CS, Lodge DM (2001) Progress in invasion biology: predicting invaders. Trends Ecol Evol 16:199-204

Kumar S, Stecher G, Li M, Knyaz C, Tamura K (2018) MEGA X: molecular evolutionary genetics analysis across computing platforms. Mol Bio Evol 35:1547-1549

Kuyulu A, Genc H, Yucel S (2019) Molecular identification of Drosophila suzukii (Matsumura) (Diptera:Drosophilidae) in Canakkale Agricultural Biotechnology. Canakkale Onsekiz Mart University Faculty of Agricultura (Submitted), Graduate School of Natural and Applied Science

Landolt P, Adams T, Rogg H (2012) Trapping spotted wing drosophila, Drosophila suzukii (Matsumura)(Diptera: Drosophilidae), with combinations of vinegar and wine, and acetic acid and ethanol. J App Entomol 136:148-154
Lavrinienko A, Kesäniemi J, Watts PC, Serga S, Pascual M, Mestres F, Kozeretska I (2017) First record of the invasive pest Drosophila suzukii in Ukraine indicates multiple sources of invasion. J Pest Sci 90:421-429

Lee JC, Bruck DJ, Curry H, Edwards D, Haviland DR, Van Steenwyk RA, Yorgey BM (2011) The susceptibility of small fruits and cherries to the spotted-wing drosophila Drosophila suzukii. Pest Manag Sci 67:1358-1367

Lee JC et al (2015) Infestation of wild and ornamental noncrop fruits by Drosophila suzukii (Diptera: Drosophilidae). Ann Entomol Soc Am 108:117-129

Lee JC et al (2016) Characterization and manipulation of fruit susceptibility to Drosophila suzukii. J Pest Sci 89:771-780

Lee JC, Wang X, Daane KM, Hoelmer KA, Isaacs R, Sial AA, Walton VM (2019) Biological control of spotted-wing Drosophila (Diptera: Drosophilidae)—current and pending tactics. J Integ Pest Manag 10:13

Legge A, Orchard J, Graffham A, Greenhalg P, Kleih U (2006) The production of fresh produce in Africa for export to the United Kingdom: mapping different value chains. Natural Resource Institute, UK

Lengyel GD, Orosz S, Kiss B, Lupták R, Kárpáti Z (2015) New records and present status of the invasive spotted wing drosophila, Drosophila suzukii (Matsumura, 1931) (Diptera) in Hungary. Acta Zool Acad Sci Hung 61:73-80

Liebhold AM, Tobin PC (2008) Population ecology of insect invasions and their management. Annu Rev Entomol 53:387-408

Lux S, Ekesi S, Dimbi S, Mohamed S, Billah M (2003) Mango-Infesting Fruit Flies in Africa: Perspectives and Limitations of Biological Biological control in IPM systems. In: Neuenschwander $\mathrm{P}$, Borgemeister $\mathrm{C}$, Langewald $\mathrm{J}$ (eds) Biological control in integrated pest management systems in Africa. CABI, Wallingford, United Kingdom, pp 277-293

Mazzi D, Bravin E, Meraner M, Finger R, Kuske S (2017) Economic impact of the introduction and establishment of Drosophila suzukii on sweet cherry production in Switzerland. Insects 8:18

Mbora A, Jamnadass R, Lilles $\varnothing$ JB (2008) Growing high priority fruits and nuts in Kenya: Uses and management. World Agroforestry Centre

Nikolouli K, Sassù F, Mouton L, Stauffer C, Bourtzis K (2020) Combining sterile and incompatible insect techniques for the population suppression of Drosophila suzukii. J Pest Sci 93:647-661

Ørsted IV, Ørsted M (2019) Species distribution models of the Spotted Wing Drosophila (Drosophila suzukii, Diptera: Drosophilidae) in its native and invasive range reveal an ecological niche shift. J Appl Ecol 56:423-435

Pretty J, Bharucha ZP (2015) Integrated pest management for sustainable intensification of agriculture in Asia and Africa. Insects 6:152-182

Rombaut A, Guilhot R, Xuéreb A, Benoit L, Chapuis MP, Gibert P, Fellous S (2017) Invasive Drosophila suzukii facilitates Drosophila melanogaster infestation and sour rot outbreaks in the vineyards. R Soc Open Sci 4:170117

Rota-Stabelli O et al. (2020) Distinct genotypes and phenotypes in European and American strains of Drosophila suzukii: implications for biology and management of an invasive organism. J Pest Sci 93:77-89

Sambrook J, Russell DW (2006) Purification of nucleic acids by extraction with phenol: chloroform Cold Spring Harbor Protocols 2006:pdb. prot4455. Cold Spring Harbor, NY: Cold Spring Harbor Laboratory Press

Sebuny M Overcoming East Africa's infrastructure challenges. In: International Trade Forum, 2015. vol 2. International Trade Centre, $\mathrm{p} 32$ 
Seehausen ML et al (2020) Evidence for a cryptic parasitoid species reveals its suitability as a biological control agent. Sci Reports $10: 1-12$

Tait G et al (2018) Large-scale spatial dynamics of Drosophila suzukii in Trentino, Italy. J Pest Sci 91:1213-1224

Tamura K, Nei M (1993) Estimation of the number of nucleotide substitutions in the control region of mitochondrial DNA in humans and chimpanzees. Mol Biol Evol 10:512-526

Toxopeus J, Jakobs R, Ferguson LV, Gariepy TD, Sinclair BJ (2016) Reproductive arrest and stress resistance in winter-acclimated Drosophila suzukii. J Insect Physiol 89:37-51

Van Timmeren S, Isaacs R (2013) Control of spotted wing drosophila, Drosophila suzukii, by specific insecticides and by conventional and organic crop protection programs. Crop Prot 54:126-133

Visual Crossing (2020) Forecast and Historical Weather Data. Visual Crossing Corporation. https://www.visualcrossing.com

Vlach J (2010) Identifying Drosophila suzukii. Oregon Department of Agriculture. http://www.ipm.ucdavis.edu/PDF/PMG/SWD-IDDsuzukii.pdf. 08 August 2019

Walsh DB et al (2011) Drosophila suzukii (Diptera: Drosophilidae): Invasive pest of ripening soft fruit expanding its geographic range and damage potential. J Integ Pest Manag 2:G1-G7
Wang X-G et al (2016) Population dynamics and ecology of Drosophila suzukii in Central California. J Pest Sci 89:701-712

Wang X, Kaçar G, Daane KM (2019) Temporal Dynamics of Host Use by Drosophila suzukii in California's San Joaquin Valley: Implications for Area-Wide Pest Management. Insects 10:206

Wolf S, Zeisler C, Sint D, Romeis J, Traugott M, Collatz J (2018) A simple and cost-effective molecular method to track predation on Drosophila suzukii in the field. J Pest Sci 91:927-935

Wolf S, Boycheva-Woltering S, Romeis J, Collatz J (2020) Trichopria drosophilae parasitizes Drosophila suzukii in seven common noncrop fruits. J Pest Sci 93:627-638

Publisher's Note Springer Nature remains neutral with regard to jurisdictional claims in published maps and institutional affiliations. 